\title{
Detection of convective initiation using Meteosat SEVIRI: implementation in and verification with the tracking and nowcasting algorithm Cb-TRAM
}

\author{
D. Merk $^{1, *}$ and T. Zinner ${ }^{1}$ \\ ${ }^{1}$ Meteorologisches Institut, Ludwig-Maximilians-Universität, Theresienstr. 37, 80333 Munich, Germany \\ *now at: Leibniz-Institut für Troposphärenforschung, Permoserstr. 15, 04318 Leipzig, Germany \\ Correspondence to: D. Merk (merk@ tropos.de)
}

Received: 23 December 2012 - Published in Atmos. Meas. Tech. Discuss.: 14 February 2013

Revised: 24 June 2013 - Accepted: 27 June 2013 - Published: 6 August 2013

\begin{abstract}
In this paper a new detection scheme for convective initiation $(\mathrm{CI})$ under day and night conditions is presented. The new algorithm combines the strengths of two existing methods for detecting CI with geostationary satellite data. It uses the channels of the Spinning Enhanced Visible and Infrared Imager (SEVIRI) onboard Meteosat Second Generation (MSG). For the new algorithm five infrared (IR) criteria from the Satellite Convection Analysis and Tracking algorithm (SATCAST) and one high-resolution visible channel (HRV) criteria from Cb-TRAM were adapted. This set of criteria aims to identify the typical development of quickly developing convective cells in an early stage. The different criteria include time trends of the 10.8 IR channel, and IR channel differences, as well as their time trends. To provide the trend fields an optical-flow-based method is used: the pyramidal matching algorithm, which is part of $\mathrm{Cb}$ TRAM. The new detection scheme is implemented in $\mathrm{Cb}$ TRAM, and is verified for seven days which comprise different weather situations in central Europe. Contrasted with the original early-stage detection scheme of Cb-TRAM, skill scores are provided. From the comparison against detections of later thunderstorm stages, which are also provided by $\mathrm{Cb}-$ TRAM, a decrease in false prior warnings (false alarm ratio) from 91 to $81 \%$ is presented, an increase of the critical success index from 7.4 to $12.7 \%$, and a decrease of the BIAS from 320 to $146 \%$ for normal scan mode. Similar trends are found for rapid scan mode. Most obvious is the decline of false alarms found for the synoptic class "cold air" masses.
\end{abstract}

\section{Introduction}

Due to their hazardous impact, such as strong winds, hail or lightning thunderstorms remain a great threat to economy and society. Especially for the aviation industry the phenomenon carries a high financial risk; Mecikalski et al. (2007) and Murray (2002) stated that their annual costs related to thunderstorms exceed tens of millions of dollars. Therefore interest is high to predict thunderstorms as early and precisely as possible. Although today's numerical weather prediction (NWP) models are able to predict the likelihood for thunderstorms occurrence in a specified area reliably, it is difficult to forecast the exact time and place and path of individual thunderstorms with NWP models alone.

NWP models attempt to simulate nonlinear dynamic processes that act on short time scales and limited spatial resolution. This often makes it necessary to parameterize convective processes. Improving spatial resolution $(<4 \mathrm{~km})$ during the last years has made it possible for NWP models to treat convection explicitly. Although a more physically meaningful life cycle is reached, NWP models still do not necessarily show better point forecasts. Furthermore constraints exist because of limited computer power. Therefore it is necessary to nest high-resolution domains into lower-resolution ones (Done et al., 2004; Tang et al., 2012). Even models resolving convective processes directly require exact measurements of small-scale moisture distribution and flow kinematics. Crook (1996) showed that the initiation process of deep convection is highly dependent on the vertical moisture and temperature gradient. A shift of just $1 \mathrm{~K}$ could make the 
difference between convective initiation (CI) and no occurrence of convection.

As a result, nowcasting, i.e. the extrapolation of existing developments based on observational data, is used to predict the development and path of individual thunderstorms. Nowcasting is made possible by means of remote sensing data with good spatial and temporal coverage. Useful data are provided by radar, satellites, or lightning networks. Outside Europe and North America radar data typically lack coverage and are affected by ground echoes especially in mountainous areas. For the detection of convective cells in a very early stage - the CI - radar data is not very useful as precipitation echoes are not observable at that stage. Although additional methods exist for the detection of earlier development using radar, like the detection of convergence lines using Bragg scattering effects due to thermodynamical gradients or Rayleigh scattering due to small insects (Weckwerth and Parsons, 2006; Wilson and Mueller, 1993), satellite data are better suited for this task. Mecikalski et al. (2010) found that lead times of up to $75 \mathrm{~min}$ for thunderstorms are possible when a set of different channel criteria for geostationary satellite data is applied. An advantage of the geostationary perspective is the continuous spatial and temporal coverage of wide regions. Twelve different channels are available for the Meteosat Spinning Enhanced Visible and InfraRed Imager (SEVIRI). Image refresh rates are $15 \mathrm{~min}$ for normal scan mode (NS), and $5 \mathrm{~min}$ for rapid scan mode (RS).

Different nowcasting tools have been developed in recent years. While some concentrate on tracking of mature thunderstorms such as the Rapid Developing Thunderstorms (RDT) algorithm (Morel and Sénési, 2002) or the MAximum Spatial COrellation Tracking TEchnique (MASCOTTE) (Carvalho and Jones, 2001), others use radar data only, e.g. the convection evolution in radar products algorithm (KONvektionsentwicklung in RADarprodukten, KONRAD) (Lang, 2001) or the Radar Tracking and Monitoring (RadTRAM) algorithm (Kober and Taffener, 2009). There also exist nowcasting algorithms for the detection of CI, e.g. SATellite Convection AnalySis and Tracking (SATCAST) (Mecikalski and Bedka, 2006). In addition to the detection of later development stages, a daytime detection of convection at an early or CI stage is part of Cb-TRAM (Thunderstorm $(\mathrm{Cb})$ Tracking and Monitoring; Zinner et al., 2008; Zinner et al., 2013). Reinhardt and Dotzek (2010) investigated the quality of CI detections for both SATCAST and Cb-TRAM. They found rather high false alarm ratios (see Sect. 3.1). These can be explained by the physical characteristics of convection. In the following study, a combination of SATCAST and Cb-TRAM is conducted to merge the strengths of both methods to detect $\mathrm{CI}$ within Cb-TRAM.

Through the work described in this manuscript, Cb-TRAM is provided with a day- and nighttime detection of early convection stages. An estimate of the CI detection skill is obtained with a verification setup utilizing the detection of later stages within Cb-TRAM for NS and RS Meteosat data. The tools on which the new detection scheme is based are described in Sect. 2. The development of the new detection and verification schemes, as well as a detailed description, are presented in Sect. 3. The verification including a comparison of the existing Cb-TRAM CI detection and the new algorithm, as well as the comparison of the NS and RS data, is presented in Sect. 4. Afterwards a summary of the method and results, and a discussion of the remaining sources of uncertainty is given in Sect. 5 .

\section{Tools for the detection of convective initiation}

The new detection scheme builds on two existing algorithms for the detection of convective clouds based on geostationary satellite data. The Cb-TRAM algorithm is introduced in Zinner et al. (2008). Changes to the detection schemes are presented in Zinner et al. (2013). The SATCAST algorithm is described by Mecikalski and Bedka (2006). Further changes to this algorithm, referred to as SATCASTv2, are described by Walker et al. (2012). In the following a short overview of these two algorithms is given.

\subsection{Cb-TRAM}

$\mathrm{Cb}$-TRAM is an algorithm for the detection, tracking, and nowcasting of intense convective cells, using the data from Meteosat SEVIRI. Cb-TRAM contains three core components: (1) the derivation of a motion vector field based on the pyramidal matching algorithm, (2) the detection of convective cells at different stages of their life cycle, and (3) the tracking and nowcasting up to $60 \mathrm{~min}$ using the motion vector field. It is used in the European Union projects "RiskAware" (2004-2006) and "FLYSAFE" (2006-2009, Tafferner et al., 2008), and ongoing DLR (Deutsches Luft- und Raumfahrtzentrum, i.e. German Aerospace Center) project "Wetter und Fliegen" (Weather and Flying) (Forster and Tafferner, 2009, 2012).

The calculation of the motion vector field depends on two consecutive satellite images. From these a disparity vector field $\boldsymbol{V}$ is derived by warping one image on the other so that either the differences of the image intensities are minimized, or the local correlation is maximized. Typically, cloud motion on small scales is dominated by the large-scale flow pattern (spatial autocorrelation). This is considered by the optical flow method used in Cb-TRAM. Technically this is implemented through an analysis on different levels of spatial resolution. A first analysis on reduced horizontal resolution (large-scale motion) is successively refined in succeeding steps down to the single-pixel level. The use of this detailed motion field enhances, on the one hand, the tracking precision for small cells and, on the other hand, allows for calculation of reliable local cooling or warming trends for cloud tops as apparent trends due to advection can be removed. Generally, local time trends are calculated by subtracting the 
image at time $t$ from a warped version of the image at $t-1$. As the disparity vector fields do not only include cloud motion, but also the changes in cloud amount, it is necessary to use slightly different timesteps or other channels to obtain an advection corrected result (see Zinner et al., 2008).

Cb-TRAM discriminates convective clouds at three different development stages. Stage 1 is called "early development" or "Cl" and covers only cloud elements showing strong vertical and/or horizontal growth. These clouds are characterized by fast cloud top cooling in IR channels and increased reflectivity in the visible channels. To this end the local trend of cloud pixels in the IR10.8 and the high-resolution visible (HRV) channels are investigated. That means, the convective cell has not necessarily reached a precipitation stage to be classified as "CI".

Stage 2 uses the cooling trend in the WV6.2 channel to detect convective cells displaying "rapid development" in the upper-tropospheric region. "Mature thunderstorm" constitutes the third stage. Mature convective cells typically show a cirrus anvil and a cloud top close to the top of the troposphere (or lower-level inversions). They can even "overshoot" over these levels. Stage 3 is mainly detected by a calculation of the difference field between the WV6.2 and the IR10.8 channels. An additional criterion used therein that improves the limitation of the detection to active convective cores is the HRV channels texture (WV6.2 channel texture at nighttime). Texture is quantified by a normalized local standard deviation field.

The tracking of Cb-TRAM provides a log file containing the life cycle of individual cell objects. The tracking is based on overlap of detected cells in consecutive images. Existing cell objects at time $t-1$ are extrapolated using the disparity vector. Afterwards, the overlap with cells of time $t$ is analysed. If no overlap is detected, the cell object of time $t$ is considered a new cell object. If more than one cell object overlaps with exactly one cell object at the current timestep, a maximum overlap decision is made. Only the cell objects' life cycle that shows the maximum area overlap is continued. Other cell objects' life cycles end. If one cell object from $t-1$ overlaps with more than one object of time $t$, again maximum overlap provides the continuation of one cell life cycle and several new cell objects.

\subsection{SATCAST}

SATCAST was initially developed for the GOES 11 and 12 data, but also first efforts have been made to use Meteosat SEVIRI. The algorithm aims at providing early warnings of thunderstorms. To this end it combines three main components to detect CI. The first is a convective cloud mask interpolated to $1 \mathrm{~km}$ resolution. The second component derives mesoscale atmospheric motion vectors (AMVs), and the third investigates actual brightness temperatures and multispectral time trends. CI in SATCAST is defined as the first detection of radar reflectivities $\geq 35 \mathrm{dBZ}$ equivalent to heavy precipitation produced by convective clouds. In their study Mecikalski and Bedka (2006) investigate the precursor signals for CI, and therefore the applied criteria can be directly compared to Cb-TRAM's first stage detection.

The convective cloud mask (Berendes et al., 2008) splits the satellite scene into four different cloud types: (1) immature cumulus defined as warm clouds $\left(>-20^{\circ} \mathrm{C}\right)$ with pronounced texture (standard deviation of brightness counts); (2) thick stratus or thin cirrus that shows both little texture and warm cloud top temperatures, (3) thick cirrus, i.e. cold clouds $\left(<-20^{\circ} \mathrm{C}\right)$ with little texture; and (4) cumulonimbus $(\mathrm{Cb})$ which typically shows cold cloud top temperatures and high texture in their active centre. This classification is achieved through a series of analyses considering the typical characteristics of convective clouds in the visible channels, which are high brightness values and distinct cloud edges, the different appearance of new cumulus clouds and $\mathrm{Cb} /$ thick cirrus in the IR10.8 channel and WV6.5-IR10.8 channel difference, the different appearance of stratus and $\mathrm{Cb}$ in terms of texture.

AMVs are calculated to derive cloud top cooling trends considering the cloud advection. The algorithm that is described in Velden et al. (1997) and Velden et al. (1998) serves as a base. The algorithm derives motion vectors on synoptic scales important for assimilation of NWP models. To this end, SATCAST investigates the satellite image for distinct cloud features that could be tracked over a defined time sequence and applies a cross-correlation technique (Merrill et al., 1991) for matching these features. This method depends on high time repeat frequencies of satellite images. Changes to this algorithm were applied in order to provide motion vectors including both synoptic scale and mesoscale vectors - the latter being associated with cumulus cloud ageostrophic motions. Quality checks applied within the Velden et al. (1997) algorithm result in a loss of mesoscale, ageostrophic motion vectors. The quality checks compare the satellite-derived motion vectors with a NWP model first guess and check the spatial connection of neighboured motion vectors. Therefore motion information on smaller scales is lost. To deal with this issue the following relaxations to the original Velden et al. (1997) algorithm were applied in order to yield a denser mesoscale AMV field (Bedka and Mecikalski, 2005): reduction of the NWP firstguess constraint as subgrid motions can not be resolved reliable by the model; changes of feature selection and vector editing schemes so that horizontal resolution of feature box size and vertical resolution are increased. By this relaxation a 20 times greater number of vectors is achieved. But also erroneous vectors are included that can result in unreliable cooling or warming trends.

Assuming that past trends continue into the future, the eight interest fields (IFs) (see Table 1) are used to detect pixels with a high chance of further convective development. For the different channel values, channel differences, and derived time trends fixed thresholds are set. 
Table 1. SATCAST GOES-11 IFs for the detection of CI.

\begin{tabular}{lll}
\hline IF & criteria & threshold \\
\hline 1 & $10.7 \mu \mathrm{m} T_{B}$ & $<273 \mathrm{~K}$ \\
2 & $10.7 \mu \mathrm{m} T_{B}$ trend & $<-4 \mathrm{~K}(15 \mathrm{~min})^{-1}$ \\
3 & $10.7 \mu \mathrm{m} T_{B}$ trend & $\Delta T_{B}(30 \mathrm{~min})^{-1}$ \\
& & $<\Delta T_{B}(15 \mathrm{~min})^{-1}$ \\
4 & $10.7 \mu \mathrm{m} T_{B}$ drop below $273 \mathrm{~K}$ & within prior $30 \mathrm{~min}$ \\
5 & $(6.5-10.7) \mu \mathrm{m}$ & -10 to $-35 \mathrm{~K}$ \\
6 & $(12.0-10.7) \mu \mathrm{m}$ & -3 to $0 \mathrm{~K}$ \\
7 & $(6.5-10.7) \mu \mathrm{m}$ trend & $>3 \mathrm{~K}$ \\
8 & $(12.0-10.7) \mu \mathrm{m}$ trend & $>2 \mathrm{~K}$ \\
\hline
\end{tabular}

With IF (1) and IF (4) the special importance of the freezing level is considered. IF (2) refers to the vertical cloud growth which results in cloud top cooling. IF (3) tests for persistence of cloud growth to assure that the observed cooling is not only a random pattern. IF (5) gives information about the cloud top height relative to the troposphere, or a very dry layer in the mid- to upper troposphere. IF (7) investigates the time trend of the channel difference in IF (5). IF (6) and IF (8) are used to highlight cloud pixels that are likely to develop into a precipitating cloud (see Table 1, Bedka and Mecikalski, 2005; Mecikalski et al., 2008, 2010; Roberts and Rutledge, 2003; Mueller et al., 2003). As in Mecikalski and Bedka (2006), in order to have confidence that CI will occur, 7 out of 8 criteria per pixel have to be met.

Mecikalski et al. (2010) also investigated the use of the additional channels provided by Meteosat SEVIRI and found 21 out of 67 initially defined IR channel differences and time trends to have the least amount of redundance for the investigation of cloud depth, updraft strength and cloud-top glaciation. As only preliminary thresholds for 123 cases are used in this study and further testing with a larger data set would be required for these IFs, the Mecikalski et al. (2010) study is not used here. Only the eight original criteria listed above are considered in the following.

\section{Development of an improved detection scheme for convective initiation}

The aim of the development of this new detection scheme for $\mathrm{CI}$ is the combination of the strengths of existing detection algorithms in a way that the advance warning of strong convective cells is improved. To achieve this aim, an analysis of strengths and weaknesses is necessary first. Time trends are used for many detection criteria within SATCAST and $\mathrm{Cb}$ TRAM. As the derivation of such trends is highly dependent on the accuracy of the calculated motion fields, the quality of these vector fields is of great importance.

The AMVs in SATCAST require the existence of features that can be tracked reliably throughout a sequence of satellite images. The extension of the original AMV algorithm, which keeps track of mesoscale motion, may result in erroneous vectors which could, accordingly, lead to unreasonable cooling trends. Especially for strong vertical wind shear conditions the accuracy of AMVs seems to drop, as outlined by Mecikalski et al. (2008).

The motion vector field in Cb-TRAM is derived on a pixel basis. It is independent of trackable features. The disparity vector field in Cb-TRAM, of course, still comprises some weaknesses. The field not only includes the pure advection, but also local development. This has to be considered correctly. Nonetheless, the matching algorithm in Cb-TRAM provides pixel-by-pixel motion fields for all clouds moving in a satellite scene derived in a physically meaningful, scaledependent way.

On the other hand, the Cb-TRAM CI detection is limited to one combination of criteria including the HRV. For this reason only daytime detection is possible. While the skill of the mature-thunderstorm detection has been evaluated using lightning data (Zinner et al., 2013), a systematic evaluation of this first stage detection is pending. Contrary to this, SATCAST uses a set of IFs with several channels' information. Compared to $\mathrm{Cb}$-TRAM this approach reduces the high sensitivity and uncertainty of a decision that depends on a single field. In addition, the importance of individual IFs for CI detection in SATCAST was already investigated by Mecikalski et al. (2008). This provides a starting point for the further implementation of selected IFs into a new method.

Following from these considerations, we decided to include parts of the SATCAST-based systematic set of criteria and thresholds into Cb-TRAM as new stage 1 "CI/early development" detection scheme. This way, we aim to improve the efficiency of SATCAST criteria through the use of the $\mathrm{Cb}$-TRAM disparity vector fields for an improved derivation of time trends. An additional objective of the following work is the provision of a day- and nighttime CI detection scheme for Cb-TRAM.

\subsection{Verification method}

In order to evaluate the skill of the detection for development stage 1 (CI/early development) in Cb-TRAM a suitable verification method has to be defined.

Typically, independent observational data should be used for validation purposes. For the verification of the correct detection of CI, lightning data, radar networks (precipitation data) or satellites (cloud data) could be considered. Although CI can be accompanied by lightning and precipitation in the transition phase to mature $\mathrm{Cb}$, following our definition, $\mathrm{CI}$ usually precedes these phenomena. Due to the time shift and the related spatial shift between early signs and actual proof of convective activity, there are no data which provide a direct validation of a CI detection. Thus, it has to be evaluated considering such a shift, e.g. using a tolerance region in space and time (cf. Reinhardt and Dotzek, 2010). 
$\mathrm{Cb}$-TRAM provides an estimate of the development of CI events into more developed thunderstorms itself. It generates a connection between the stages $\mathrm{CI} /$ early development and rapid development or mature thunderstorms via its tracking capability. We decided to use this feature for verification in the following. Although collected by the same sensor, these are at least partially independent data, as the detection schemes of stage 2 and stage 3 are providing information on later stages of development using different channel combinations. Cb-TRAM's skill to detect mature-thunderstorm clouds is evaluated in Zinner et al. (2013): the probability of detecting a mature intense convective cell is about $77 \%$, at least during the day. At the same time only about $16 \%$ of all stage 3 detections do not show any convective activity in terms of lightning. Similar to the verification in Zinner et al. (2013) an object-based verification method is applied here, using the cell objects generated by Cb-TRAM and the related life cycle log data for each cell. If the Cb-TRAM's CI detections are perceived as forecasts of further development into thunderstorms, and stages 2 and 3 for the related $\mathrm{Cb}$-TRAM object as proof of convective development, then one can define the following categorical variables regarding the development of each individual cell object:

- A hit $H$ is a cell object at stage 1 that shows further development into stage 2 or 3 within $60 \mathrm{~min}$.

- A false alarm $F$ is a cell object at stage 1 that does not show further development within $60 \mathrm{~min}$.

- A miss $M$ is a cell object at stage 2 or 3 without any stage 1 detection during the previous $60 \mathrm{~min}$.

This definition is rather ambitious as the evaluation is done on an individual cell basis instead of an evaluation of the general affinity of a wide tolerance region to display further thunderstorm development. This has to be taken into account when comparing our values to less strictly defined verifications (e.g. Reinhardt and Dotzek, 2010; Mecikalski et al., 2008). Nonetheless, this definition complies with common sense and thus provides appreciable result values. It is well suited to compare the two CI algorithms within Cb-TRAM, and the results can be directly provided by the Cb-TRAM algorithm.

Different verification statistics can be calculated Roebber (2009) with these categorical variables. In this paper the following are used: POD (probability of detection), FAR (false alarm ratio), CSI (critical success index), BIAS.

The ideal value is $100 \%$ for the POD, CSI, and BIAS. It is $0 \%$ for the FAR. POD and FAR should be considered as a pair. It is possible to improve the POD by just randomly increasing the number of forecast objects, but this would normally result in an synchronous increase of the FAR. The POD provides the fraction of correctly detected early developments when a thunderstorm followed the detection, while the FAR provides the fraction of detections which are not followed by thunderstorms. The CSI combines both, the number of hits and the number of false alarms. Typically the CSI shows small values for rare events, like CI or thunderstorms in general, as the number of hits is low. The BIAS simply gives the ratio of forecasted to observed number of events. Values of the BIAS above $100 \%$ constitute overforecasting, and below $100 \%$ under-forecasting. Nonetheless the BIAS does not judge how well observations and forecasts correspond.

A Cb-TRAM cell object can represent several consecutive cell life cycles of a multi-cell thunderstorm. This is because the tracking algorithm will allocate a new development, close enough to the expected track, to an already existing cell in a decaying stage. As a result more than one CI classification per cell object is possible. Consider the following example object history: $0 \mathrm{~min}$ - stage 3 detection (mature); $15 \mathrm{~min}$ - stage 3; $30 \mathrm{~min}$ - stage 3; $45 \mathrm{~min}$ - stage 1 (CI); $60 \mathrm{~min}$ - stage 2 (rapid development); and $75 \mathrm{~min}$ - stage 3. This means that the cell life cycle starts with a missed CI development, but nonetheless shows a hit at $45 \mathrm{~min}$.

\subsection{Convective cloud IF mask}

The first step of the new detection scheme is the limitation to an IF mask for convective clouds within a full satellite scene. Similar to SATCAST's convective cloud mask only pixels within this IF mask are evaluated further. This step aims at the reduction of false alarms that can be found in areas where the likelihood for convective clouds in the satellite image scence is minimal. The IF mask is derived using three different tests:

- $253 \mathrm{~K}<\mathrm{IR} 10.8<278 \mathrm{~K}$

- HRV reflectivity $>0.5$ (can be used only under daytime conditions)

- Local standard deviation at WV7.3 and IR10.8 larger than defined threshold.

The first test investigates the cloud top temperature in the IR10.8 channel. Clouds with top temperatures $<253 \mathrm{~K}$ typically have reached higher altitudes so that it is likely for those clouds to be either $\mathrm{Cb}$ or thick cirrus. It is very unlikely for CI to show top temperatures below this threshold value. The same threshold is used within SATCAST to separate mature from initial convective clouds. Cloud top temperatures above $278 \mathrm{~K}$ refer to very low clouds, such as cumulus or stratus. SATCAST uses $273 \mathrm{~K}$ as one typical criterion for CI. This value is relaxed here to account for a wider range of convective developments.

Test two is adapted from Cb-TRAM. Only those pixels with a reflectivity higher than the given threshold are considered for CI. Convective clouds typically are bright due to their high optical thickness. Lower values of reflectivity are most likely caused by thinner clouds or scattered cloudiness in a given pixel (e.g. mostly very small cumulus). 


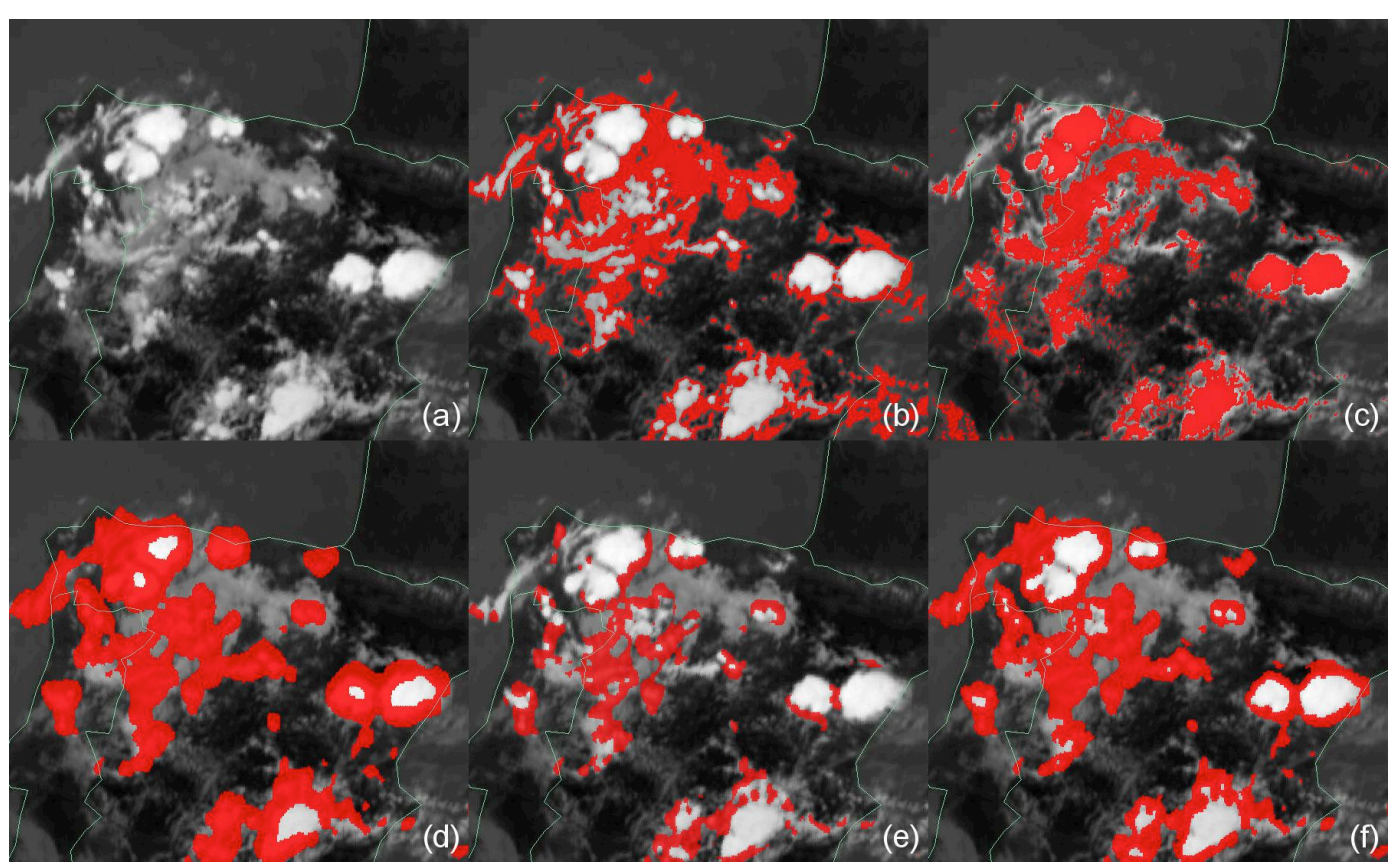

Fig. 1. Limitation to IF: (a) IR10.8 cloud scene over Iberian Peninsula with various (convective) cloud types. Different tests for the convective cloud IF masks: (b) $253 \mathrm{~K}<\mathrm{IR} 10.8<278 \mathrm{~K}$, (c) HRV $>0.5$, (d) local standard deviation at WV7.3 and IR10.8, (e) IF mask at daytime, (f) and nighttime condition (without HRV). Positive test results for each pixel are plotted in red.

A distinct signal of cumulus clouds that is taken into account for the third test is their lumpy appearance. The convective process does not produce a smooth cloud top structure, which is more likely for, e.g., cirrus and stratus. Although the HRV channel would provide the best horizontal resolution to detect such variability, the IR10.8 and WV7.3 are used here to provide a method applicable day and night. While the IR10.8 channel gives the possibility of detecting all clouds in the lower troposphere (if no overlaying clouds in the upper layer exists), the WV7.3 is used to guarantee that clouds have reached a significant altitude at a lower midtroposphere level (approx. $3000 \mathrm{~m}$ ). The localized standard deviation (cf. Zinner et al., 2008) is calculated for a local area around each pixel using a Gaussian kernel. Threshold values are derived by mean values of the detected CI objects within the existing Cb-TRAM version. Separate masks of these three tests are presented in Fig. 1 for a case over the Iberian Peninsula together with the resulting IF for both dayand nighttime conditions.

\subsection{Scoring system}

As for SATCAST a scoring system is adopted for a set of criteria for CI detection with a final decision based on the number of met criteria. A basic set of six criteria is selected from SATCAST by means of a statistical analysis and some general considerations. For MSG SEVIRI, channel IR10.8 is used instead of IR10.7 onboard GOES, and WV6.2 instead of WV6.5. Criteria with proven values for CI detection are prefered (i.e. the eight SATCAST GOES criteria). For these criteria tested threshold values exist and can be used immediately. An additional criterion is the processing time requirement as a nowcasting tool, of course, should provide results quickly. Thus the number of utilized channels and subsequently detection criteria is limited to a minimum while preserving the best possible result. Beforehand some of the SATCAST IF thresholds have been modified. For IF 2 the IR10.8 cooling rate is set to $-6 \mathrm{~K}$ within $15 \mathrm{~min}$ (compare Tables 1 and 2). Reasonable cooling rates for CI detection following Roberts and Rutledge (2003) are between -4 and $-8 \mathrm{~K}$ within $15 \mathrm{~min}$ for weak and strong growth rates, respectively. The time frame in IF 3 for which the temperature dropped below the freezing point is set to $15 \mathrm{~min}$. Roberts and Rutledge (2003) found out that $15 \mathrm{~min}$ after this criteria is fulfilled, first convective radar echoes can be observed.

In analogy to the study by Mecikalski et al. (2008), an analysis for different criteria combinations was performed for an independent training data set, including three days in summer (9 June 2009, 3 July 2009, 19 July 2009) from 07:00 to 17:00 UTC. The eight SATCAST criteria (Table 1) and the existing $\mathrm{Cb}$-TRAM criterion are used in combination with the convective cloud IF mask. The criteria are split into four groups with different physical basis to limit the combination of needed tests (Table 2). Group 1 describes the IR cooling trend, Group 2 the relative height to the tropopause and Group 3 the split window channel test with the individual time trends. Group 4 is the Cb-TRAM detection for CI, investigating HRV brightening and IR10.8 cooling. The statistical 


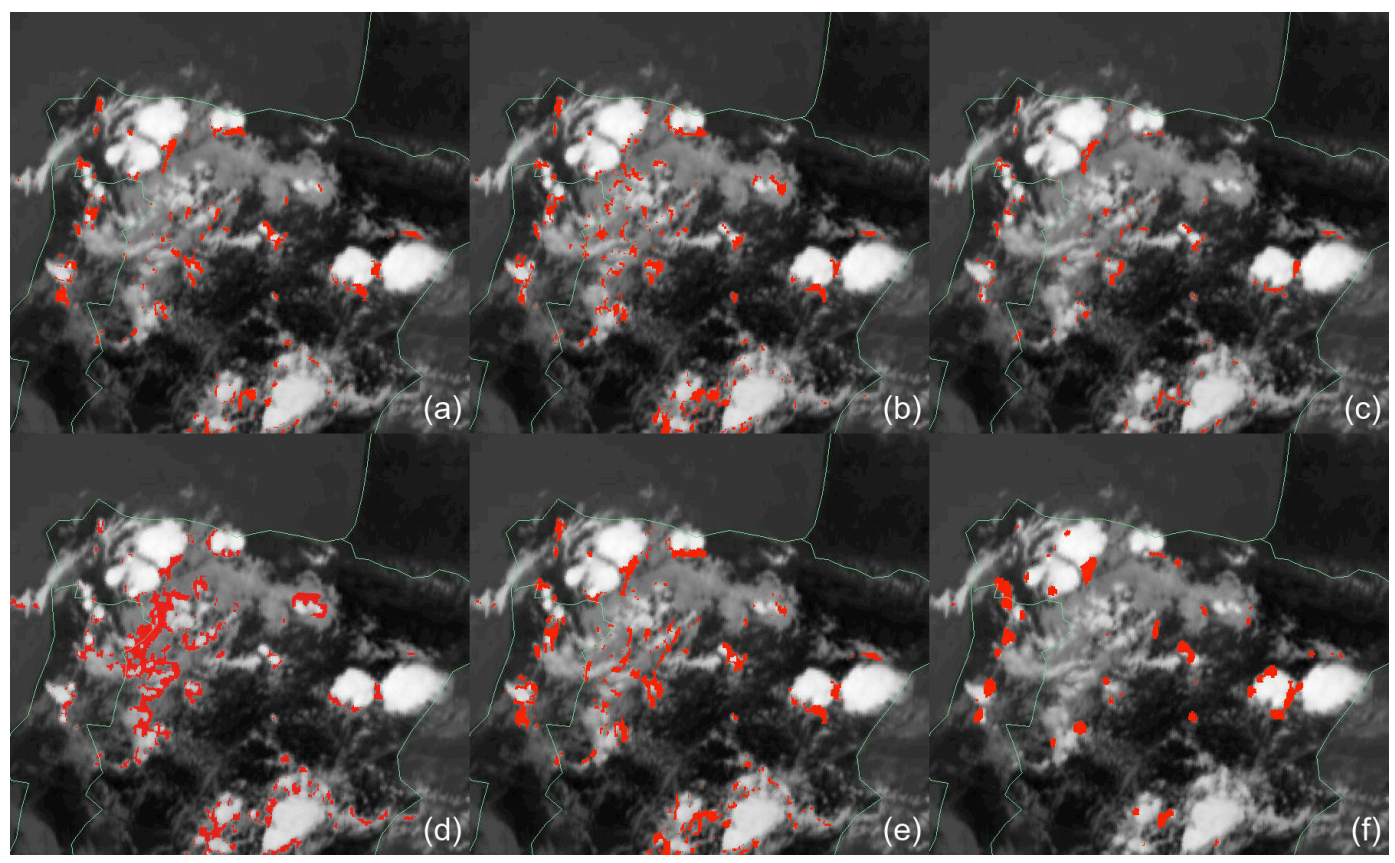

Fig. 2. Different masks for the six separate criteria listed in Table 3 for the same scene as in Fig. 1. Pixels plotted in red meet the individual thresholds for the defined criterion. (a) Criterion \#1: 15 min IR10.8; (b) \#2: 30 min IR10.8; (c) \#3: freezing level IR10.8; (d) \#4: WV6.2IR10.8; (e) \#5: 15 min WV6.2-IR10.8; (f) \#6: HRV × IR10.8.

Table 2. Grouped set of criteria for CI detection.

\begin{tabular}{|c|c|}
\hline Criteria & Critical value \\
\hline \multicolumn{2}{|c|}{ Group 1 (IR10.8 cooling trend) } \\
\hline $\begin{array}{l}10.8 \mu \mathrm{m} T_{B} \text { trend } \\
10.8 \mu \mathrm{m} T_{B} \text { trend } \\
T_{B} \text { in } 10.8 \mu \mathrm{m} \\
\text { drop below } 273 \mathrm{~K}\end{array}$ & $\begin{array}{l}<-6 \mathrm{~K}(15 \mathrm{~min})^{-1} \\
\Delta T_{B}(30 \mathrm{~min})^{-1}<\Delta T_{B}(15 \mathrm{~min})^{-1} \\
\text { within last } 30 \mathrm{~min}\end{array}$ \\
\hline \multicolumn{2}{|c|}{ Group 2 (relative height within troposphere and growth) } \\
\hline $\begin{array}{l}(6.2-10.8) \mu \mathrm{m} \\
(6.2-10.8) \mu \mathrm{m} \text { trend }\end{array}$ & $\begin{array}{l}-10 \text { to }-35 \mathrm{~K} \\
>3 \mathrm{~K}\end{array}$ \\
\hline \multicolumn{2}{|c|}{ Group 3 (split window channel test) } \\
\hline $\begin{array}{l}(12.0-10.8) \mu \mathrm{m} \\
(12.0-10.8) \mu \mathrm{m} \text { trend }\end{array}$ & $\begin{array}{l}-3 \text { to } 0 \mathrm{~K} \\
>2 \mathrm{~K}\end{array}$ \\
\hline \multicolumn{2}{|c|}{ Group 4 (Cb-TRAM CI detection) } \\
\hline $\mathrm{HRV} \times 10.8 \mu \mathrm{m}$ & Cb-TRAM threshold \\
\hline
\end{tabular}

analysis is described in detail in Sect. 3.1. Similar to the study of Mecikalski et al. (2008), 15 possible combinations of the four groups were investigated. The best trade-off concerning CSI and FAR was yielded by the combination of group 1, group 2 and group 4 . The criteria in group 3 aim to detect clouds that are already glaciated and therefore are in a very final phase of CI. We decided to omit these criteria for our purposes. This is due to the slightly different emphasis on early development and, consequently, the fact that such development is already covered by the stage 2 detection within $\mathrm{Cb}$-TRAM, and the result of the combinations. Finally the set of six different criteria from groups 1, 2 and 4 as listed in Table 3 is used to further investigate the pixels in the previously derived IF mask. An example of the individual criteria is given in Fig. 2.

At daytime conditions, five of six criteria have to be met at a given time for a given pixel to consider this pixel to show CI. At nighttime the five remaining IR criteria have to be met (without the criterion 6). Further a minimum object size is required. Only CI pixels which have at least two neighbouring pixels are kept. This is analogous to the other Cb-TRAM detection schemes and is mainly used to avoid false alarms related to very small short-lived objects.

\section{Skill of CI detection: verification within Cb-TRAM}

In the following section the verification method described in Sect. 3.1 is applied on NS and RS Meteosat SEVIRI data for a representative number of days. The resulting skills are always presented in comparison to the original stage 1 detection scheme in Cb-TRAM for a clearer appreciation. 
Table 3. Set of six criteria used for the new CI detection algorithm together with the individual thresholds for NS and RS.

\begin{tabular}{llll}
\hline$\#$ & Criteria & Critical value (NS) & Critical value (RS) \\
\hline 1 & $10.8 \mu \mathrm{m} T_{B}$ time trend & $<-6 \mathrm{~K}(15 \mathrm{~min})^{-1}$ & $<-2 \mathrm{~K}(5 \mathrm{~min})^{-1}$ \\
2 & $10.8 \mu \mathrm{m} T_{B}$ time trend & $\Delta T_{B}(30 \mathrm{~min})^{-1}<\Delta T_{B}(15 \mathrm{~min})^{-1}$ & $\Delta T_{B}(15 \mathrm{~min})^{-1}<\Delta T_{B}(10 \mathrm{~min})^{-1}<\Delta T_{B}(5 \mathrm{~min})^{-1}$ \\
3 & $10.8 \mu \mathrm{m} T_{B}$ drop below $273 \mathrm{~K}$ & within last $15 \mathrm{~min}$ & within last $15 \mathrm{~min}$ \\
4 & $(6.2-10.8) \mu \mathrm{m}$ & -10 to $-30 \mathrm{~K}$ & -10 to $-30 \mathrm{~K}$ \\
5 & $(6.2-10.8) \mu \mathrm{m}$ time trend & $>3 \mathrm{~K}$ & $>1 \mathrm{~K}$ \\
6 & $\mathrm{HRV} \times 10.8 \mu \mathrm{m}$ & Cb-TRAM NS threshold & Cb-TRAM RS threshold \\
\hline
\end{tabular}
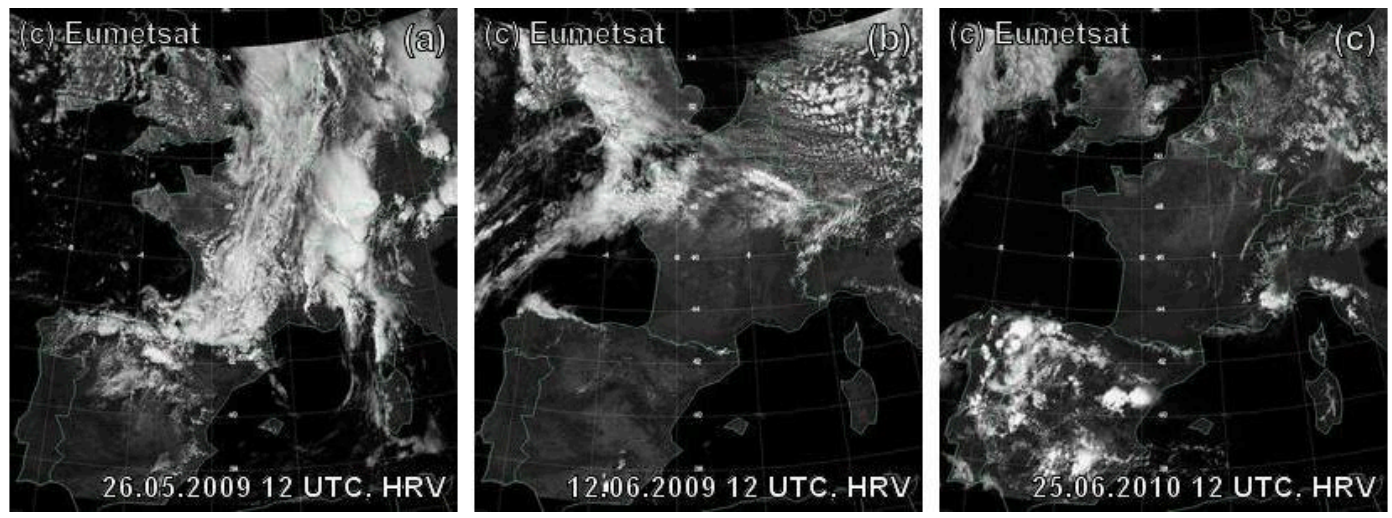

Fig. 3. High-resolution visible (HRV) Meteosat images representative for the synoptic classes (a) "cold front" on 26 May 2009 , (b) "cold air" on 12 June 2009, (c) "high pressure" on 25 June 2010, 12:00 UTC.

\subsection{Test cases and conditions}

The verification is carried out for seven different test days over the area of central Europe. These days can be classified into three different, typical synoptic weather conditions for thunderstorm development:

- class "cold front": convection connected with a coldfront passage. The typical pattern is an upper-level trough over the west coast of the Atlantic, passing over the area of interest during the observation period. Convection is triggered directly at the frontal region and in the prefrontal moist and destabilized air mass. Prefrontal high convective available potential energy (CAPE) values can be observed, and also deep layer shear is present. 26 May 2009 (day 1), 6 June 2010 (day 2), 3 July 2010 (day 3).

- class "cold air": advection of cold air masses together with an active upper-level low and presence of surface heating leads to instability and triggering of many convective cells. Both thunderstorm and non-electric rain showers occur in a typical honeycombed structure. 14 July 2010 (day 4) and 12 June 2009 (day 5).

- class "high pressure": convection connected with weakforcing conditions. A typical pattern is an upper-level ridge stretching over central Europe, connected to a low-level high-pressure area, generally suppressing cloud formation because of subsidence. Convection is mainly triggered orographically or by sufficient surface heating to overcome Convective INhibition (CIN). 25 June 2010 (day 6) and 29 June 2010 (day 7).

Representative satellite images for the three classes are given in Fig. 3. For all test scenarios the same configuration in Cb-TRAM is used. The analysed area lies between -9.5 and $11.5^{\circ}$ longitude and 36.5 and $55.5^{\circ}$ latitude. As the area investigated covers central Europe, for some of these days a clear distinction of synoptic regimes is not easy. The classification is done by addressing the most dominant synoptic feature of the individual test days. For example, the 14 July 2010 day shows convection along the cold front and convection triggered by advection of cold air behind cold fronts. The latter feature is more dominant (more convective cells) here. Thus, this day is classified as a cold-air case. In Fig. 4 an example comparison between the current and the new CI detection is given for this test case on 14 July 2010. For evaluation of daytime conditions the time frame between 07:00 and 17:00 UTC is used. For nighttime conditions two individual time frames of 00:00 to 07:00 UTC and 17:00 to 23:45 UTC (23:55 UTC for RS mode) are used for each day. For 29 June 2010 the RS data sets are incomplete over a longer time period. This day is therefore not included in the statistics. 


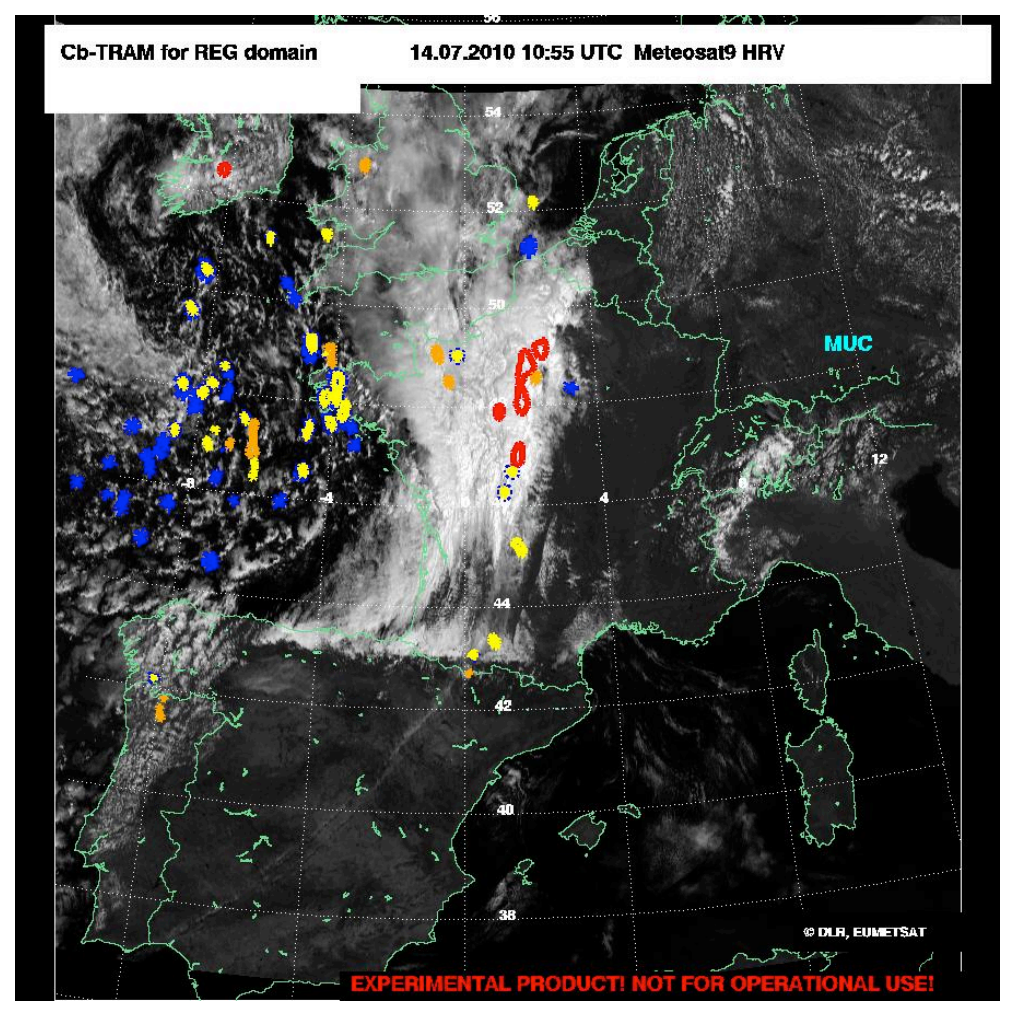

Fig. 4. Example of the test domain and Cb-TRAM for 14 July 2014. Yellow structures are CI objects detected by the new algorithm; blue structures are objects detected by the previous algorithm. Red and orange are the rapid-development and mature Cb objects, respectively.

\subsection{Verification results}

\subsubsection{Normal scan mode}

The results for daytime conditions in NS mode are investigated as total values over all seven test days, as sub-totals for the three synoptic conditions, and for each test day individually. This is done to get an overall impression of the new detection algorithm compared to the existing one, and to explore the behaviour under different synoptic weather conditions. The total values as listed in Table 4 show an increase in hits by 4 using the new algorithm instead of the existing one, a decrease in false alarms by 3108 , and an increase in misses by 84 . This manifests an improvement, but has to be put into perspective. POD, FAR, CSI and BIAS, also shown in Fig. 5, all reflect this improvement.

The striking charcteristic of both CI detections, as well as CI detection skill in general, is obviously a large FAR around $90 \%$ (and relatedly a large BIAS) while the POD is $45 \%$ at best. At this point, it must be emphasized that this is, on the one hand, owed to the choice of rather conservative verification definitions (Sect. 3.1); on the other hand, it is inherent to the involved physics. Usually a single thunderstorm's strong updraft is preceded by a number of early less confined convective developments. Our verification method allows only an allocation of one CI object, which consists of neighbouring pixels, to one thunderstorm object. From the whole area that shows signs of early development, usually only one object in the near surrounding is selected by very localized characteristics. The preferred CI object will soon dominate all other CI objects in the surrounding, as low-level convergence and upper-level divergence suppress other updrafts. Consequently a large number of false alarms has to be expected. The mediocre POD is in part simply owed to the multi-cell nature of most storms in the analysis. Early development of a secondary cell is often masked by the preceding older cell's later life cycle stages.

The percentage of thunderstorms that are preceded by a previous detection of CI, i.e. receive an advance warning, shows only a small change from 29 to $28 \%$ over all days (POD). The most striking improvement is a nearly halved absolute number of false alarms $(5427 \rightarrow 2319)$. The FAR decreases from 91 to $81 \%$. For the reasons mentioned above, this is still a high absolute number, and is reflected in the clear over-forecasting tendency (BIAS still larger 146\%). Nonetheless, in the framework of different development detections in Cb-TRAM this makes sense. Although the specific CI detection might not carry a high probability of developing further, still an accumulation of CI detection in certain regions should be regarded as an indication that $\mathrm{CI}$ is imminent. 


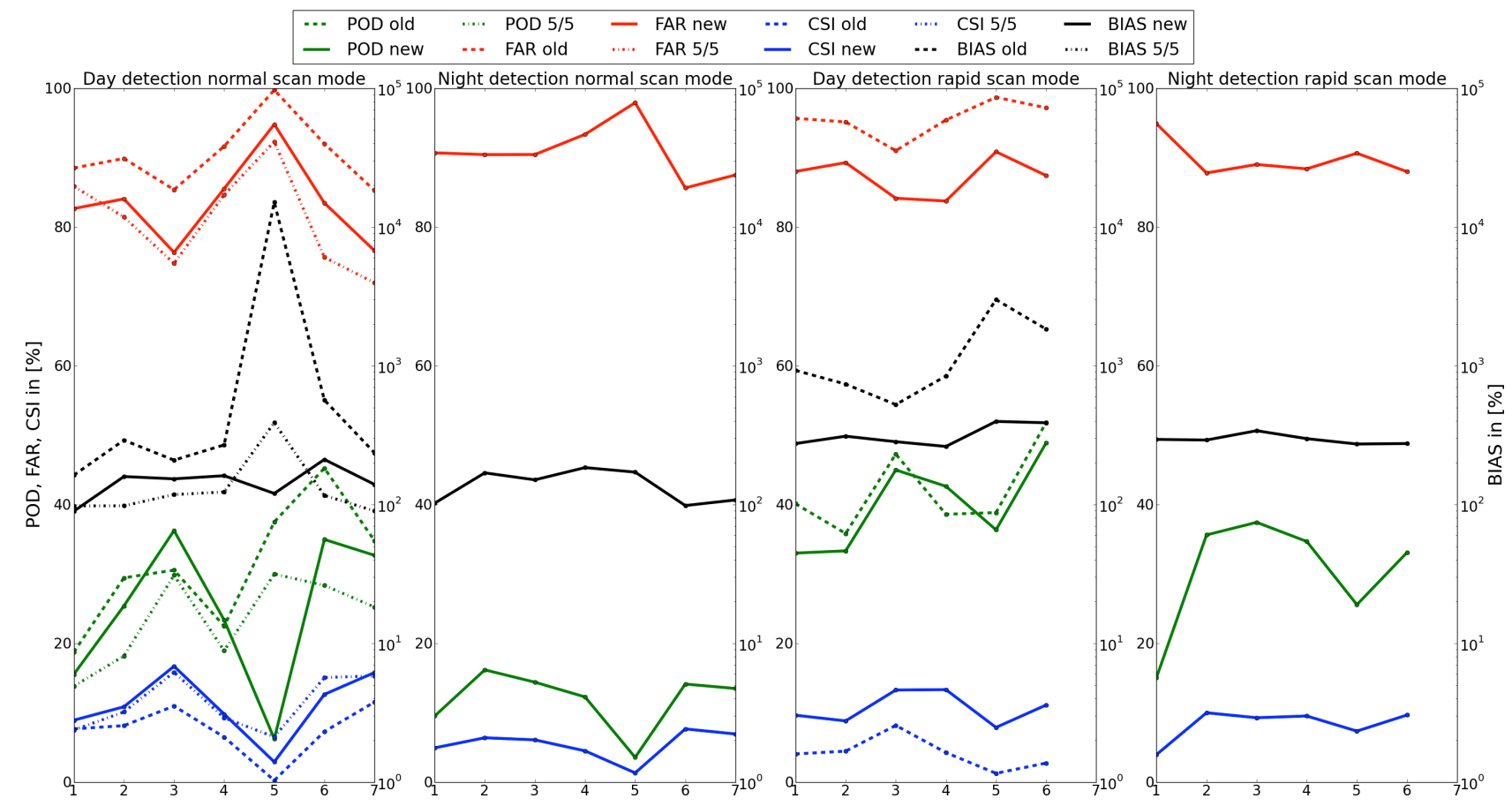

Fig. 5. POD, FAR, CSI and BIAS for the test days: green, red, blue and black lines, respectively. Dashed lines mark the original CI detection algorithm results, while the bold lines mark the new CI detection results; dotted lines in the left figure mark the new nighttime detection mode applied to the daytime data (using the five IR criteria only).

The CSI, combining $F, H$ and $M$, increases from 7 to $13 \%$, a significant near doubling of CSI. This improvement in the overall forecasting behaviour also is reflected in the decrease of the over-forecasting tendency from 320 to $146 \%$. Therefore, although still noisy, a clearer signal of potentially dangerous convective developments is provided by the new algorithm.

In the day-by-day analysis in Fig. 5 it can be seen that best day's FAR is around $76 \%$ and POD around $45 \%$, with worst day's FAR at $95 \%$ and POD at $15 \%$. BIAS does show a peak value at 12 June 2009 for both the new and previous detection scheme, while it does not vary much for the rest of the days. The newer method shows a higher or equal number of hits on 4 out of 7 days compared to the original CI detection, and a lower number of false alarms for all seven days. This results in a better FAR and CSI for all days and increased POD on 3 days. In order to take a closer look at the individual synoptic conditions, we have calculated total values for each synoptic class. In the following the individual day results are summarized first; afterwards a closer look at characteristics influences on the individual days is taken.

- A visual inspection (not shown) gives following typical cloud structures: for the class of cold-front days there is a high amount of clouds visible, both convective (especially $\mathrm{Cb}$ ) and non-convective (cirrus or stratus). Therefore the satellite perspective on $\mathrm{CI}$ along the front is limited (e.g. due to prevailing cirrus shields of mature $\mathrm{Cb}$ ). Typical for the class of cold-air days is a honeycombed structure of many convective cells. Geostationary satellites provide a good view on CI that is very widespread under these unstable atmospheric conditions. For the class of high-pressure days fewer clouds are visible compared to the other two classes. Convection mainly depends on the time of day.

- For the grouped statistical values for each of the synoptic classes, the highest POD is found for high-pressure cases, both for the previous and new detection algorithm. The greatest increase for the new algorithm is found for high-pressure cases, followed closely by coldair cases. FAR in the previous algorithm is lowest for cold-front cases. A possible explanation for this, at the first moment surprising result, is the more persistent dynamic convection trigger mechanism. This way the statistically random nature of the CI process is reduced. For the new algorithm, the FAR is lowest at highpressure cases, followed closely by cold-front cases. The greatest decrease in FAR is achieved for highpressure days. For the new algorithm, the best CSI values are found for high pressure, followed by cold-front cases. The lowest BIAS value is found for cold-front cases. The highest reduction of BIAS compared to the original algorithm is found for cold-air cases (by nearly one third), where the influence of the 12 June 2009 case is most striking. 
Table 4. Categorical variables and statistics under daytime (07:00-17:00 UTC) conditions for the old and the new algorithm (old $\rightarrow$ new). Listed are the results for NS and RS mode for each of the test days as well as the results for the three synoptic classes. Hits, misses and false alarms are given as absolute numbers; POD, FAR, CSI and BIAS are expressed as a percentage. For each of the synoptic classes the average (av.) values are given.

\begin{tabular}{|c|c|c|c|c|c|c|c|c|}
\hline $\mathrm{Nr}$ & Date & Hits & False alarms & Misses & POD (in \%) & FAR (in \%) & CSI (in \%) & BIAS (in \%) \\
\hline \multicolumn{9}{|c|}{ Normal scan mode } \\
\hline \multirow{2}{*}{\multicolumn{2}{|c|}{$\begin{array}{l}\text { all days (total) } \\
\text { cold front (av.) }\end{array}$}} & $538 \rightarrow 542$ & $5427 \rightarrow 2319$ & $1329 \rightarrow 1413$ & $28.8 \rightarrow 27.7$ & $91.0 \rightarrow 81.0$ & $7.4 \rightarrow 12.7$ & $320 \rightarrow 146$ \\
\hline & & $85 \rightarrow 90$ & $598 \rightarrow 359$ & $250 \rightarrow 262$ & $25.4 \rightarrow 25.6$ & $87.6 \rightarrow 80.0$ & $9.1 \rightarrow 12.7$ & $204 \rightarrow 128$ \\
\hline 1 & 26 May 2009 & $80 \rightarrow 69$ & $616 \rightarrow 329$ & $347 \rightarrow 375$ & $18.7 \rightarrow 15.5$ & $88.5 \rightarrow 82.7$ & $7.7 \rightarrow 8.9$ & $163 \rightarrow 89$ \\
\hline 2 & 6 June 2010 & $53 \rightarrow 48$ & $471 \rightarrow 253$ & $127 \rightarrow 141$ & $29.4 \rightarrow 25.4$ & $89.9 \rightarrow 84.0$ & $8.1 \rightarrow 10.9$ & $291 \rightarrow 159$ \\
\hline \multirow[t]{2}{*}{3} & 3 July 2010 & $121 \rightarrow 153$ & $707 \rightarrow 494$ & $275 \rightarrow 269$ & $30.6 \rightarrow 36.3$ & $85.4 \rightarrow 76.4$ & $11.0 \rightarrow 16.7$ & $209 \rightarrow 153$ \\
\hline & old air (av.) & $34 \rightarrow 36$ & $960 \rightarrow 238$ & $115 \rightarrow 115$ & $22.9 \rightarrow 23.8$ & $96.6 \rightarrow 86.9$ & $3.1 \rightarrow 9.3$ & $667 \rightarrow 182$ \\
\hline 4 & 14 July 2010 & $65 \rightarrow 68$ & $713 \rightarrow 402$ & $224 \rightarrow 223$ & $22.5 \rightarrow 23.4$ & $91.6 \rightarrow 85.5$ & $6.5 \rightarrow 9.8$ & $269 \rightarrow 162$ \\
\hline 5 & 12 June 2009 & $3 \rightarrow 4$ & $1206 \rightarrow 73$ & $5 \rightarrow 6$ & $37.5 \rightarrow 40.0$ & $99.8 \rightarrow 94.8$ & $0.2 \rightarrow 4.8$ & $15113 \rightarrow 770$ \\
\hline \multicolumn{2}{|c|}{ high pressure (av.) } & $78 \rightarrow 100$ & $857 \rightarrow 384$ & $176 \rightarrow 200$ & $30.7 \rightarrow 33.3$ & $91.7 \rightarrow 79.3$ & $7.0 \rightarrow 14.6$ & $368 \rightarrow 161$ \\
\hline 6 & 25 June 2010 & $81 \rightarrow 64$ & $933 \rightarrow 323$ & $98 \rightarrow 119$ & $45.3 \rightarrow 35.0$ & $92.0 \rightarrow 83.5$ & $7.3 \rightarrow 12.7$ & $567 \rightarrow 212$ \\
\hline 7 & 29 June 2010 & $135 \rightarrow 136$ & $781 \rightarrow 445$ & $253 \rightarrow 280$ & $34.8 \rightarrow 32.7$ & $85.3 \rightarrow 76.6$ & $11.5 \rightarrow 15.8$ & $236 \rightarrow 140$ \\
\hline \multicolumn{9}{|c|}{ Rapid scan mode } \\
\hline \multirow{2}{*}{\multicolumn{2}{|c|}{$\begin{array}{l}\text { all days (total) } \\
\text { cold front (av.) }\end{array}$}} & $1599 \rightarrow 2064$ & $35754 \rightarrow 1846$ & $2141 \rightarrow 2987$ & $42.8 \rightarrow 40.9$ & $95.7 \rightarrow 86.2$ & $4.1 \rightarrow 11.5$ & $999 \rightarrow 295$ \\
\hline & & $348 \rightarrow 396$ & $5244 \rightarrow 2506$ & $466 \rightarrow 614$ & $42.8 \rightarrow 39.2$ & $93.8 \rightarrow 86.4$ & $5.7 \rightarrow 11.3$ & $687 \rightarrow 287$ \\
\hline 1 & 26 May 2009 & $280 \rightarrow 273$ & $6191 \rightarrow 2004$ & $418 \rightarrow 554$ & $40.1 \rightarrow 33.0$ & $95.7 \rightarrow 88.0$ & $4.1 \rightarrow 9.6$ & $927 \rightarrow 275$ \\
\hline 2 & 6 June 2010 & $191 \rightarrow 215$ & $3742 \rightarrow 1791$ & $342 \rightarrow 430$ & $35.8 \rightarrow 33.3$ & $95.1 \rightarrow 89.3$ & $4.5 \rightarrow 8.8$ & $737.9 \rightarrow 311$ \\
\hline 3 & 3 July 2010 & $573 \rightarrow 701$ & $5799 \rightarrow 3722$ & $639 \rightarrow 857$ & $47.3 \rightarrow 45.0$ & $91.0 \rightarrow 84.2$ & $8.2 \rightarrow 13.3$ & $526 \rightarrow 284$ \\
\hline \multicolumn{2}{|c|}{ cold air (av.) } & $173 \rightarrow 327$ & $6395 \rightarrow 1893$ & $438 \rightarrow 457$ & $38.7 \rightarrow 41.7$ & $97.4 \rightarrow 85.3$ & $2.5 \rightarrow 12.2$ & $1469 \rightarrow 283$ \\
\hline 4 & 14 July 2010 & $244 \rightarrow 565$ & $5093 \rightarrow 2912$ & $388 \rightarrow 760$ & $38.6 \rightarrow 42.6$ & $95.4 \rightarrow 83.8$ & $4.3 \rightarrow 13.3$ & $845 \rightarrow 262$ \\
\hline 5 & 12 June 2009 & $101 \rightarrow 88$ & $7696 \rightarrow 874$ & $159 \rightarrow 154$ & $38.8 \rightarrow 36.4$ & $98.7 \rightarrow 90.9$ & $1.3 \rightarrow 7.9$ & $2999 \rightarrow 398$ \\
\hline \multicolumn{2}{|c|}{ high pressure (av.) } & $210 \rightarrow 222$ & $7233 \rightarrow 1543$ & $195 \rightarrow 232$ & $51.9 \rightarrow 48.9$ & $97.2 \rightarrow 87.4$ & $2.8 \rightarrow 11.1$ & $1838 \rightarrow 389$ \\
\hline 6 & 25 June 2010 & $210 \rightarrow 222$ & $7233 \rightarrow 1543$ & $195 \rightarrow 232$ & $51.9 \rightarrow 48.9$ & $97.2 \rightarrow 87.4$ & $2.8 \rightarrow 11.1$ & $1838 \rightarrow 389$ \\
\hline
\end{tabular}

- A closer look at the 12 June 2009 case shows convective cells over large parts of central Europe as a result of instability due to advection of cold air masses and surface heating, with the typical honeycomb structure of convective cells, but nearly no further development to stage 2 or 3 occurred during the whole day. The original Cb-TRAM CI detection generates many false alarms and only three hits. The latter are connected with some scattered cells in Spain. The new detection algorithm detects one more hit, but it shows a drastic 16-fold decrease in false alarms. In this case it is obvious that the small number of hits distorts the significance of POD and FAR on this day. Although the number of false alarms decreases by 1133 , i.e. decisively, the FAR only decreases by $5 \%$.

- The other day in this test sample which is worth considering more closely is 3 July 2010 . This day provides a rather untypical increase in hits, compared to the other cold front and even all days evaluated. A visual inspection shows CI primarily at a convergence line ahead of the front and a lot of CI objects over the Alps. An unobstructed satellite view on these developments together with the sustained lifting mechanism leads to many successful CI detections. The main difference to the original detection scheme seems to be the improved detection of orographically induced CI. This results in an increased number of hits, but also a reduction of false alarms.

- Although the synoptic classes are arranged by their dominant similarities, some special features can occur on individual days. The reduction of false alarms is especially high on the cold-air day of 12 June 2009 and the two high-pressure days, with the false alarms reduced to the third on 25 June 2010. A visual inspection shows that the eastern part of the domain on the latter day is also influenced by a cold-core low.

Summarizing for cases with cold air masses, the criteria of the new CI detection algorithm result in a drastically reduced number of false alarms. But false alarms also decreased on the other test days by a significant amount.

For nighttime conditions no direct comparison is possible due to the fact that the original CI algorithm did not provide detections at night. Therefore only the new results are investigated, again both for all test nights and for each night individually (see Table 5). In addition the nighttime detection skill can be evaluated by an application to the daytime 


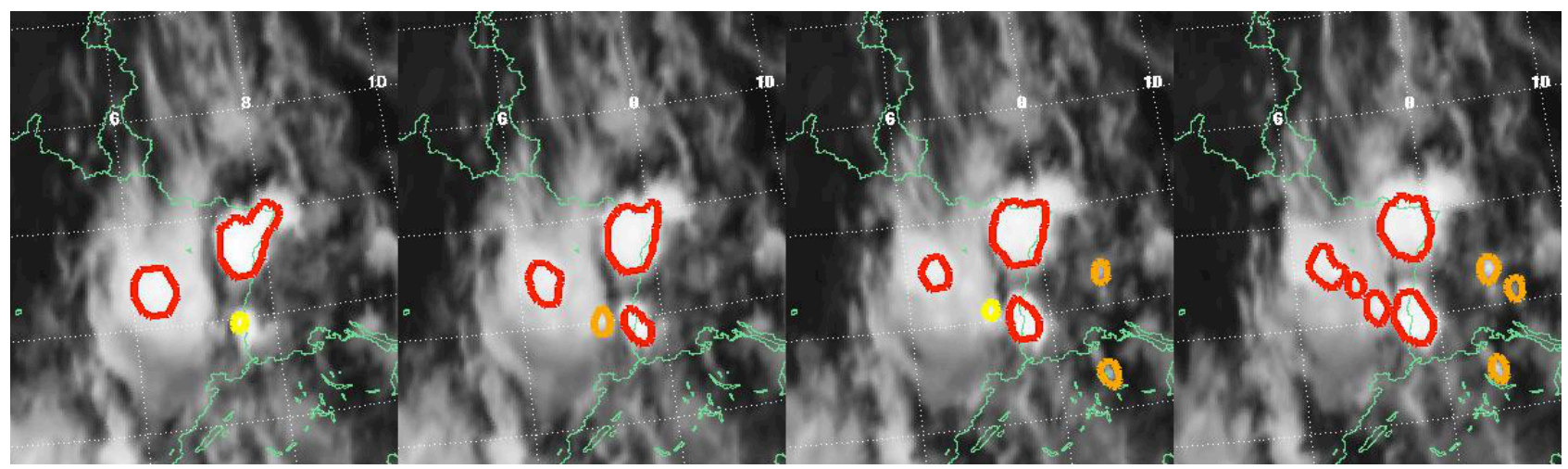

Fig. 6. Development of new thunderstorm cells in an orographic environment over the Black Forest and Jura Mountains in the night of 3 July 2010 between 22:30 and 23:15 UTC. The plots show the cell objects as polygons in the same colours as in Cb-TRAM (yellow: CI; orange: rapid development; red: mature thunderstorm) on top of the IR10.8 satellite images.

data; i.e. only the the five IR criteria are used. As can be seen in Fig. 5, there is only a slight difference between day- and nighttime detection for FAR, CSI and BIAS compared to the improvements achieved by the new method. The main difference lies in a reduction in POD (excluding day 5), as expected due to the missing HRV information, but at the same time the FAR is slightly reduced also.

At nighttime over land a general decrease of convective activity is observed (as in this investigation). Missing radiation and a stable boundary layer result in unfavourable conditions for the formation of convection. Due to the lower activity at nighttime the values of POD, FAR and CSI show poorer values compared to daytime as early developments (CI) tend to "die out" over night. Newly forming convective cells are interrupted in their development cycle at nightfall if no dynamic trigger is active. This results in increased number of false alarms.

The total results over all test nights (00:00-07:00 UTC and 17-00:00 UTC) show a POD of $13 \%$, a FAR of $90 \%$ and a CSI of $6 \%$. The POD, CSI and FAR values for most days are very similar, except day 5 . This day shows a very high FAR and a low POD. This can be explained by the very small number of cases in these values. Nearly no convective activity occurs during this night due to missing forcing. Consequently the number of false alarms is the smallest of all nights, too. In the high-pressure cases a visual inspection provides the trend of decreasing CI activity after sunset. Although this is the case, referring to the best POD and FAR values for highpressure situations, these seem to give the best possibility of detecting remaining cells. Considering the longer time frame for night (14h), and that activity at the onset of night may be still higher than later at night, the tendency of decreasing activity at nighttime can still be seen by the smaller number of hits and misses (representing the total active cells). In our set of test days also some days with nighttime forcing are present. An example of a cell development at nighttime observed with the new detection algorithm in Cb-TRAM is given in Fig. 6. New convective cells over the Black Forest and the Jura Mountains develop after sunset. In general, the CI detection at nighttime is most promising when synoptic dynamic or orographic triggers are present.

\subsubsection{Rapid scan mode}

In SEVIRI RS mode, the repeat cycle for new satellite images is $5 \mathrm{~min}$ instead of $15 \mathrm{~min}$. Therefore a three times better temporal coverage is reached compared to NS. It seems obvious that this should improve the possibility of detecting and tracking especially rapidly changing cloud processes such as CI. This should therefore manifest in a higher number of detected hits. In the following a comparison of the results of the RS detections of CI using the original and the new detection scheme is presented first. The basis is six of the seven test days presented for the NS analysis before. Data for 29 June 2010 shows too many gaps and is therefore omitted. The eventual benefits of RS versus NS are explored afterwards.

For RS mode some slight modifications in the detection algorithms were made (see Table 3 ) to account for the better time resolution. These include (1) investigating trend values in IF 1 and IF 5 over the last $5 \mathrm{~min}$ instead of last $15 \mathrm{~min}$ using adapted threshold values for this shorter time frame; (2) sustained cooling in IF 2 is considered for the last 15 instead of $30 \mathrm{~min}$; and (3) Cb-TRAM RS threshold for IF 6 is used. For NS mode at least $30 \mathrm{~min}$ ( 2 timesteps) have to be considered for sustained cooling criteria, while for RS we can use shorter intervals. Roberts and Rutledge (2003) found that after $15 \mathrm{~min}$ the first precipitation is observed under sustained cooling conditions. In this way, setting IF 2 to $15 \mathrm{~min}$ provides a physically meaningful combination of the two criteria for detecting strong convective cells in an early stage. Verification is done using the same $60 \mathrm{~min}$ time frame for further developments of cells, but now given the chance to verify this using 5 min timesteps. The total number of cell objects 
Table 5. Categorical variables and statistics under nighttime (00:00-07:00 UTC, 17:00-00:00 UTC) conditions for the new algorithm. Hits, misses and false alarms are given as absolute numbers; POD, FAR, CSI and BIAS are expressed as a percentage. For each of the synoptic classes the average (av.) values are given.

\begin{tabular}{|c|c|c|c|c|c|c|c|c|}
\hline \multicolumn{9}{|c|}{ Normal scan mode } \\
\hline $\mathrm{Nr}$ & Date & Hits & False alarms & Misses & POD (in \%) & FAR (in \%) & CSI (in \%) & BIAS (in \%) \\
\hline- & all days & 164 & 1543 & 1076 & 13.2 & 90.4 & 5.9 & 138 \\
\hline- & cold front (av.) & 31 & 295 & 198 & 13.5 & 90.5 & 5.9 & 142 \\
\hline 1 & 26 May 2009 & 20 & 195 & 190 & 9.5 & 90.7 & 5.0 & 102 \\
\hline 2 & 6 June 2010 & 39 & 369 & 202 & 16.2 & 90.4 & 6.4 & 169 \\
\hline 3 & 3 July 2010 & 34 & 322 & 202 & 14.4 & 90.5 & 6.1 & 151 \\
\hline- & cold air (av.) & 11 & 171 & 89 & 11.0 & 94.0 & 4.1 & 182 \\
\hline 4 & 14 July 2010 & 21 & 295 & 150 & 12.3 & 93.4 & 4.5 & 185 \\
\hline 5 & 12 June 2009 & 1 & 47 & 27 & 3.6 & 98.0 & 1.3 & 171 \\
\hline- & high pressure (av.) & 25 & 158 & 153 & 14.0 & 86.3 & 7.4 & 103 \\
\hline 6 & 25 June 2010 & 27 & 161 & 164 & 14.1 & 85.6 & 7.7 & 98 \\
\hline 7 & 29 June 2010 & 22 & 154 & 141 & 13.5 & 87.5 & 6.9 & 108 \\
\hline \multicolumn{9}{|c|}{ Rapid scan mode } \\
\hline- & all days (total) & 1275 & 10968 & 2795 & 31.3 & 89.6 & 8.5 & 301 \\
\hline- & cold front (av.) & 266 & 2375 & 589 & 31.1 & 89.9 & 8.2 & 309 \\
\hline 1 & 26 May 2009 & 96 & 1786 & 542 & 15.0 & 94.9 & 4.0 & 295 \\
\hline 2 & 6 June 2010 & 383 & 2755 & 692 & 35.6 & 87.8 & 10.0 & 292 \\
\hline 3 & 3 July 2012 & 319 & 2585 & 533 & 37.4 & 89.0 & 9.3 & 341 \\
\hline- & cold air (av.) & 173 & 1405 & 372 & 31.7 & 89.0 & 8.9 & 290 \\
\hline 4 & 14 July 2010 & 256 & 1948 & 482 & 34.7 & 88.4 & 9.5 & 299 \\
\hline 5 & 12 June 2009 & 89 & 862 & 259 & 25.6 & 90.6 & 7.4 & 273 \\
\hline- & high pressure (av.) & 141 & 1032 & 285 & 33.1 & 88.0 & 9.7 & 275 \\
\hline 6 & 25 June 2010 & 141 & 1032 & 285 & 33.1 & 88.0 & 9.7 & 275 \\
\hline
\end{tabular}

increases due to the larger number of timesteps investigated, and more short-living convective storms can be observed.

Under daytime conditions the sum for all six RS test days shows an increase of hits by 465 , a decrease of false alarms by 22908 (relative decrease of more than 60\%) and an increase of misses, comparing original and new algorithm. The increase of misses is a technical side effect which results from an increased number of stage 2 and 3 cell objects. Although the detection of stage 2 and 3 itself is not modified, changes of stage 1 detection influence the stage 2 and 3 objects. Each object includes all neighbouring pixels with all earlier detection stages. Missing stage 1 pixels formerly connecting stage 2 and 3 pixels lead to a breaking up of these stage 2 and 3 objects into more individual objects. This results in a higher amount of misses. This issue is more pronounced in RS mode, but also is observed in NS mode. This effect leads to the slight decrease of POD on 4 of 6 days although the number of hits increases on 5 days. FAR decreases from 96 to $86 \%$, CSI increases from 4 to $11.5 \%$, BIAS decreases from 999 to $295 \%$. The number of hits increases for 4 out of the 6 days, while it shows a decrease on 2 days. False alarm numbers are improved for all 6 days. On 12 June 2009 only about $10 \%$ of the original false alarms remain. Best values of POD (around 50\% for RS) were found for the previous and new algorithm under high-pressure conditions. With almost a doubling of hits, the biggest increase in POD is yielded for cold-air cases here. Applying the new detection method, a decrease of POD for the two other classes is observed. This is due to the discussed technical issue resulting in more misses. Best FAR values for the previous algorithm are found for cold-front cases. Applying the new algorithm, best FAR values are found for the cold-air cases. The improvement of FAR for the cold-air cases is the best of all three classes, comparing the old and new algorithm. The same is true for CSI values. This supports the NS results, where the FAR improved mainly for the cold air mass conditions as well. In Fig. 5 it is obvious that the overall behaviour of FAR, POD, CSI and BIAS does not show any major differences between day and night.

In the following, we address the question of whether the use of RS data improves the detection skill over the use of NS data. As we apply some of the criteria from Mecikalski and Bedka (2006) to 5min data with adjusted thresholds (Table 3), there is the chance to observe rapidly developing cells. The higher time resolution further allows evaluating the development over three timesteps within the last 15 min. Short-living cells that may be missed by using only 15 min timesteps are detected this way. Even with the overall 
increase of detectable cells in RS mode, we achieved a lower BIAS compared to the previous algorithm in NS mode. A forecaster using the new algorithm will get a clearer picture of the situation updated every $5 \mathrm{~min}$. For the new algorithm compared between NS and RS, BIAS is a factor of 2 higher in RS mode. This is due to the higher number of hits and the higher chance of providing false alarms. We observe higher POD values for all three synoptic classes in RS compared to NS mode. FAR and CSI are only better for the cold-air class. With the drastically decreasing number of false alarms and the related reduction of tracking processes for these cells, we also obtain an improvement in processing time. This is an important point for a nowcasting tool providing real-time warnings.

\section{Conclusions}

By combination of the strengths of two existing detection algorithms (Cb-TRAM by Zinner et al., 2008, and SATCAST by Mecikalski and Bedka, 2006) for geostationary satellite data, a new detection scheme for CI has been developed and implemented in Cb-TRAM. A set of criteria from the SATCAST CI detection, using thermal IR channel measurements for one point in time as well as a derived time trend, has been combined with detailed cloud motion and deformation fields from the Cb-TRAM pyramidal matching algorithm. In the latter an optical-flow-based method is of special importance for the derivation of detailed cloud top cooling trends. A pixel-by-pixel analysis of six different CI tests using the channels WV6.2 and IR10.8, and during daytime including the HRV became possible this way. In order to reduce the number of false detections decisively, an additional preselection of convective clouds with an IF mask excludes all areas with non-convective clouds.

The convective cloud IF mask is limited to clouds which are cold, bright, and show enough small-scale texture (in IR10.8, in HRV, and in WV7.3 and IR10.8, respectively). On this IF mask, the set of six CI criteria aims to assess the typical signatures of quickly developing convective cells at an early stage, such as strong updrafts resulting in strong cloud top cooling and increasing cloud top height.

Two criteria use IR10.8 time trends over different periods of time. The drop of temperature below freezing level is analysed, as well as the value of the difference between WV6.2 and IR10.8 and their time trend. Finally the original $\mathrm{Cb}$-TRAM criterion for $\mathrm{CI}$ is included which requires simultaneous brightening in the HRV and cooling in the IR10.8. A scoring system connects different criteria: pixels are considered to display $\mathrm{CI}$ in a satellite scene if five out of six possible criteria are met during daytime; at nighttime all five IR criteria have to be met.

For the analysis of the skill of the original and the newly developed methods, a verification of the CI detections against later stages of the convective life cycle has been used. For that purpose the further development to the subsequent life cycle stages "rapid cooling" and "mature thunderstorm" within Cb-TRAM has been investigated. Cb-TRAM cell objects which show further development after CI detection within 60 min are considered a hit, missing further development a false alarm, and a missing CI detection within $60 \mathrm{~min}$ before further development a miss. By means of POD, FAR, CSI, and BIAS seven test days and nights are analysed for NS and six for RS METEOSAT SEVIRI data.

The main results are as follows:

- FAR for NS mode under daytime conditions decreases from 91 to $81 \%$, and CSI increases from 7.4 to $12.7 \%$, while BIAS is reduced from 320 to $146 \%$, using the new detection algorithm instead of the original $\mathrm{Cb}$ TRAM method. Thus an improvement for all these statistical verification values is reached. POD decreases slightly although the number of detected hits are increasing. This is due to a technical side effect concerning detection of misses in Cb-TRAM.

- Considering each day individually FAR and CSI show improvement for each day, POD for three out of seven days.

- The CI detection newly implemented in Cb-TRAM shows a reduction of false alarms for all test days, both for NS (15 min data) and RS ( $5 \mathrm{~min}$ ), when compared to the original detection.

- The decline in false alarms is most prominent for synoptic conditions within cold-core lows that produce a lot of convective cells in NS and RS mode.

- An important improvement within Cb-TRAM is the fact that the new algorithm works during day and night: POD, FAR and CSI at night are of the same order of magnitude as during the day. A comparison of day- (including HRV criterion) and nighttime detection (only IR criteria) during the daytime hours reveals mainly an increase of POD through the use of the HRV information.

Generally, the high values of FAR for most of the days lead to the question on the limitations of the detection of CI using geostationary data only, and their use as an early warning for stronger convective storm development. The statistical character of convection has to be considered. Only a small part of a large number of CI candidate cumuli will develop into mature thunderstorms. The most intense cell will suppress surrounding development through its impact on surface convergence and upper-level divergence. In addition, not each cumulus cloud satisfying the typical criteria for fast growth over some time develops into a $\mathrm{Cb}$ during its life cycle. Unfavourable conditions for further development, such as the interruption of a sufficient supply of warm moist air, a stable layer at some height above the convective cloud top, or the advection of the whole cell into an area with unfavourable 
conditions for convection, could stop the development at any time of the cell life cycle.

Together with the choice of the verification method, this all sets narrow boundaries for the quality values possible. Since the verification is done by investigating the further development of each CI object against the detections for rapiddevelopment and mature-thunderstorm objects within $\mathrm{Cb}$ TRAM, the results also depend on the quality of these detections. For $77 \%$ of all mature-thunderstorm objects, an overlap with lightning was found in Zinner et al. (2013). Some CI cell objects within an area of rapidly growing cumulus clouds may be classified as false alarms in a conservative verification setup. Here we track each object's development history individually. It could be equally legitimate to classify a whole area of multiple CI objects as hits if only one single strong thunderstorm develops out of the area. Walker et al. (2012) implemented an object-tracking method based on Zinner et al. (2008) and verified the cell objects against radar data. Taking into account all of the cell objects, he received a POD value of $32 \%$ comparable to our outcome. The FAR of $55 \%$ is lower than in our study but has to be put into perspective. Walker et al. (2012) use a manual search in the surrounding area which is less strict than our automated tracking.

While the number of hits did increase only slightly for some of the days, the decline of false alarms is the clearest improvement as far as the comparison to the original CI detection within Cb-TRAM is concerned. As shown, this decrease is more pronounced for days classified as cold air. These days strongly influence the total values for all days. The omission of tracking false alarm cells also results in an improvement of calculation time. The calculated statistical values of POD, FAR and CSI and BIAS depend on the numbers of hits, misses and false alarms. Especially for a low total number of observed objects these skill values tend to be very sensitive to small changes of the latter categorial variables.

Calculations for different synoptic groups depend on the possibility of distinguishing synoptic patterns for the whole domain. Although this was possible for most of the days used here, mixed situations occur (e.g. 14 July 2010). Longer time series would be desirable to corroborate the results found in this study.

The detection of CI in general is highly dependent on cloud top cooling trends. These, on the other hand, rely on the disparity vector field. Advection of optically thin cirrus clouds over cumulus clouds can lead to apparent cloud top cooling values which are not an effect of rising cloud tops. Although the presented method uses an optical flow method, incorrect vectors within the field can obviously not be ruled out. This may lead to situations where false alarms are diagnosed which are actually only an effect of erroneous cooling trends and not thunderstorms.

Due to the geostationary position of Meteosat satellites, the best horizontal resolution is available at the equator, decreasing with higher latitudes. For Europe this results in a reduced horizontal resolution by a factor of about 1.5. The actual resolution for HRV is about $1.5 \mathrm{~km} \times 1.5 \mathrm{~km}$ and $4 \mathrm{~km} \times 6 \mathrm{~km}$ for the standard-resolution Meteosat channels for Europe. Therefore few details are visible which are of particular importance for small-scale developments like CI.

The oblique viewing angle around $50^{\circ}$ also affects the observation of cloud tops. More and more cloud side information influences the measured signal and the derived cloud top temperatures. Therefore the thresholds suggested by Mecikalski and Bedka (2006) may be slightly shifted for higher latitudes. Using strict thresholds does also not account for developments just below these given limits. Thus some hits can be missed.

Considering the difficulties arising when detecting and verifying CI, this leads to the question of how these could be further improved. Investigating microphysical properties for convective clouds at/near the cloud top using satellite data as done by Mecikalski et al. (2011) could improve the understanding of in-cloud processes during convection and therefore help to find typical patterns that can be used for CI detection. Application of stability data fields provided by satellites or NWP model outputs may help to reduce the amount of false alarms. One can also think of other strategies to combine different criteria for CI detection, e.g. using fuzzy logic as in Cb-TRAM. Another approach for verification could be to soften the object cell perspective and verify whole areas where CI takes place.

Acknowledgements. EUMETSAT and DLR Oberpfaffenhofen are acknowledged for providing SEVIRI data. We thank Dennis Stich for the fruitful discussions. We thank two anonymous reviewers for improving this paper. This manuscript is dedicated to the memory of our colleague Hermann Mannstein, who died unexpectedly and much too early on 25 January 2013. He was a scientific innovator behind many cloud remote sensing activities at DLR over nearly three decades. Among other things, he developed the Cb-TRAM core algorithms. He will be deeply missed as expert, advisor and friend.

Edited by: S. Malinowski

\section{References}

Bedka, K. M. and Mecikalski, J. R.: Application of SatelliteDerived Atmospheric Motion Vectors for Estimating Mesoscale Flows, J. Appl. Meteorol., 44, 1761-1772, 2005.

Berendes, T. A., Mecikalski, J. R., MacKenzie, W. M., Bedka, K. M., and Nair, U. S.: Convective cloud identification and classification in daytime satellite imagery using standard deviation limited adaptive clustering, J. Geophys. Res., 113, D20207, doi:10.1029/2008JD010287, 2008.

Carvalho, L. and Jones, C.: A satellite method to identify structural properties of mesoscale convective systems based on the maximum spatial correlation tracking technique (MASCOTTE), J. Appl. Meteorol., 40, 1683-1701, 2001. 
Crook, N. A.: Sensitivity of moist convection forced by boundary layer processes to low-level thermodynamic fields, Mon. Weather Rev., 124, 1768-1785, 1996.

Done, J., Davis, C. A., and Weisman, M.: The next generation of NWP: explicit forecasts of convection using the weather research and forecasting (WRF) model, Atmos. Sci. Lett., 5, 110-117, doi:10.1002/asl.72, 2004.

Forster, C. and Tafferner, A.: An integrated user-oriented weather forecast system for air traffic using real-time observations and model data, in: Proceedings of the European Air and Space Conference (CEAS), 26-29 October 2009, Manchester, UK, 2009.

Forster, C. and Tafferner, A.: Nowcasting Thunderstorms for Munich Airport, DLR-Forschungsbericht, Deutsches Zentrum für Luft- und Raumfahrt e.V., Bibliotheks- und Informationswesen, Köln, 2012.

Kober, K. and Taffener, A.: Tracking and nowcasting of convective cells using remote sensing data from radar and satellite, Meteorol. Z., 1, 075-084, 2009.

Lang, P. (Ed.): Cell tracking and warning indicator derived from operational radar products, Proceedings of the 30th International Conference on Radar Meteorology, Munich, Germany, 2001.

Mecikalski, J. R. and Bedka, K. M.: Forecasting Convective Initiation by Monitoring the Evolution of Moving Cumulus in Daytime GOES Imagery, Mon. Weather Rev., 134, 49-78, 2006.

Mecikalski, J. R., Berendes, T. A., Feltz, W. F., Bedka, K. M., Bedka, S. T., Murray, J. J., Wimmers, A. J., Minnis, P., Johnson, D. B., Haggerty, J., Bernstein, B., Pavolonis, M., and Williams, E.: Aviation Applications for Satellite-Based Observations of Cloud Properties, Convection Initiation, In-Flight Icing, Turbulence, and Volcanic Ash, B. Am. Meteorol. Soc. 88, 1589-1607, doi:10.1175/BAMS-88-10-1589, 2007.

Mecikalski, J. R., Bedka, K. M., Paech, S. J., and Litten, L. A.: A Statistical Evaluation of GOES Cloud-Top Properties for Nowcasting Convective Initiation, Mon. Weather Rev., 136, 48994914, 2008.

Mecikalski, J. R., MacKenzie Jr., W. M., Koenig, M., and Muller, S.: Cloud-Top Properties of Growing Cumulus prior to Convective Initiation as Measured by Meteosat Second Generation, Part I: Infrared Fields, J. Appl. Meteorol. Clim., 49, 521-534, 2010.

Mecikalski, J. R., Watts, P. D., and Koenig, M.: Use of Meteosat Second Generation optimal cloud analysis fields for understanding physical attributes of growing cumulus clouds, Atmos. Res., 102, 175-190, 2011.

Merrill, R. T., Menzel, W. P., Baker, W., Lynch, J., and Legg, E.: A report on the recent demonstration of NOAA's upgraded capability to derive satellite cloud motion winds, B. Am. Meteorol. Soc., 72, 372-376, 1991.

Morel, C. and Sénési, S.: A climatology of mesoscale convective systems over Europe using satellite infrared imagery, I: Methodology, Q. J. Roy. Meteorol. Soc., 128, 1953-1971, 2002.

Mueller, C. K., Saxen, T., Roberts, R., Wilson, J., Betancourt, T., Dettling, S., Oien, N., and Yee, J.: NCAR Auto-Nowcast system, Weather Forecast., 18, 545-561, 2003.
Murray, J. J.: Aviation weather applications of Earth Science Enterprise data, Earth Observ. Mag., 11, 27-30, 2002.

Reinhardt, B. and Dotzek, N.: Validation of the MSG Convection Initiation Product (Final Report), Tech. rep., EUMETSAT Contract EUM/CO/09/4600000642/MK, available from EUMETSAT, 2010.

Roberts, R. D. and Rutledge, S.: Nowcasting Storm Initiation and Growth Using GOES-8 and WSR-88D Data, Weather Forecast., 18, 562-584, 2003.

Roebber, P. J.: Visualizing multiple measures of forecast quality, Weather Forecast., 24, 601-608, 2009.

Tafferner, A., Forster, C., Senesi, S., Guillou, Y., Laroche, P., Delannoy, A., Lunnon, B., Turb, D., Hauf, T., and Markovic, D.: Nowcasting thunderstorm hazards for flight operations: the $\mathrm{Cb}$ WIMS approach in FLYSAFE, in: Proc. 26th Int. Congress of the Aeronautical Sci. (ICAS), 14-19 September 2008, Anchorage, Alaska, 2008.

Tang, Y., Lean, H. W., and Bornemann, J.: The benefits of the Met Office variable resolution NWP model for forecasting convection, Meteorol. Appl., doi:10.1002/met.1300, in press, 2012.

Velden, C. S., Hayden, C. M., Nieman, S. J., Menzel, W. P., Wanzong, S., and Goerss, J. S.: Upper-Tropospheric Winds Derived from Geostationary Satellite Water Vapor Observations, B. Am. Meteorol. Soc., 78, 173-195, doi:10.1175/15200477(1997)078<0173:UTWDFG > 2.0.CO;2, 1997.

Velden, C. S., Olander, T. L., and Wanzong, S.: The Impact of Multispectral GOES-8 Wind Information on Atlantic Tropical Cyclone Track Forecasts in 1995, Part I: Dataset Methodology, Description, and Case Analysis, Mon. Weather Rev., 126, 1202-1218, doi:10.1175/1520-0493(1998)126<1202:TIOMGW>2.0.CO;2, 1998.

Walker, J. R., MacKenzie, W. M., Mecikalski, J. R., and Jewett, C. P.: An Enhanced Geostationary Satellite-Based Convective Initiation Algorithm for 0-2-h Nowcasting with Object Tracking, J. Appl. Meteorol. Clim., 51, 1931-1949, doi:10.1175/JAMC-D11-0246.1, 2012.

Weckwerth, T. M. and Parsons, D. B.: A Review of Convection Initiation and Motivation for IHOP2002, Mon. Weather Rev., 134, 5-22, 2006.

Wilson, J. W. and Mueller, C. K.: Nowcasts of Thunderstorm Initiation and Evolution, Weather Forecast., 8, 113-131, 1993.

Zinner, T., Mannstein, H., and Tafferner, A.: Cb-TRAM: Tracking and monitoring severe convection from onset over rapid development to mature phase using multi-channel Meteosat-8 SEVIRI data, Meteorol. Atmos. Phys., 101, 191-210, 2008.

Zinner, T., Forster, C., de Coning, E., and Betz, H.-D.: Validation of the Meteosat storm detection and nowcasting system Cb-TRAM with lightning network data - Europe and South Africa, Atmos. Meas. Tech., 6, 1567-1583, doi:10.5194/amt-6-1567-2013, 2013. 